\title{
Kybernetika
}

\section{Jiřina Vejnarová}

On decision-making in possibility theory

Kybernetika, Vol. 51 (2015), No. 3, 457-468

Persistent URL: http://dml.cz/dmlcz/144380

\section{Terms of use:}

(C) Institute of Information Theory and Automation AS CR, 2015

Institute of Mathematics of the Czech Academy of Sciences provides access to digitized documents strictly for personal use. Each copy of any part of this document must contain these Terms of use.

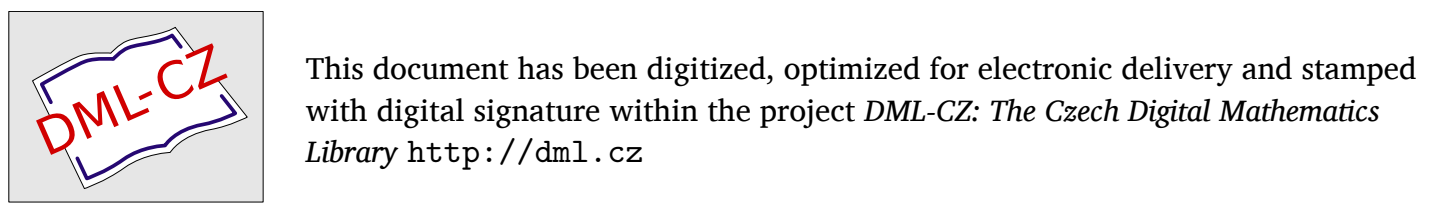




\title{
ON DECISION-MAKING IN POSSIBILITY THEORY
}

\author{
JIŘINA VEJNAROVÁ
}

We present an alternative approach to decision-making in the framework of possibility theory, based on the idea of decision-making under uncertainty. We utilize the fact, that any possibility distribution can be viewed as an upper envelope of a set of probability distributions to which well-known minimax principle is applicable. Finally, we recall also an alternative to the minimax rule, so-called local minimax principle. Local minimax principle not only allows sequential construction of decision function, but also appears to play an important role exactly in the framework of possibility theory due to its sensitivity. Furthermore, the optimality of a decision function is easily verifiable.

Keywords: possibility measures and distributions, upper envelopes of probability distributions, decision functions, minimax principle

Classification: $91 \mathrm{~B} 06,62 \mathrm{C} 20$

\section{INTRODUCTION}

The problem of decision-making arising from everyday practice belongs to the oldest problem types studied by mathematicians from 17th century. Three types of decisionmaking are recognized: under certainty, under risk and under uncertainty. While the first type is rather simple and (from the mathematical viewpoint) uninteresting, the remaining two have been studied from different points of view. During this period several optimality principles were adopted, as e.g. Bayesian, maximum-likelihood or minimax principles.

However, the emergence of new mathematical tools as alternatives to probability theory during the last fifty years opened a lot of new problems. Probability theory, nevertheless, has often served as a source of inspiration for the development of these calculi. Paper [5] by Ivan Kramosil belongs to works of this kind. He presented a formal possibilistic analogy of both Bayesian optimal and minimax decision functions in a highly abstract framework of lattice-valued possibility measures.

In this paper, we will also deal with decision-making in possibility theory, however we adopt totally different approach. We will utilize the fact that any possibility distribution can be associated with a set of probability distributions dominated by it and find an optimal decision function in possibility theory using results from probabilistic framework.

DOI: $10.14736 / \mathrm{kyb}-2015-3-0457$

This is a modified version of the original paper to reflect a correction of an editorial error. 
The paper is organized as follows: after a brief overview of basic concepts from possibility theory (Section 2) and decision-making in probabilistic framework (Section 3) in Section 4 we will introduce possibilistically optimal decision function and in Section 5 locally possibilistically optimal decision function and study their relationship.

\section{BASIC CONCEPTS}

The purpose of this section is to give an overview of basic concepts and notation concerning possibility measures and distributions and credal sets dominated by them.

\subsection{Possibility measures and distributions}

Let $\mathbf{X}$ be a finite set called universe of discourse which is supposed to contain at least two elements. A possibility measur $\oint^{1} \Pi$ is a mapping from the power set $\mathcal{P}(\mathbf{X})$ of $\mathbf{X}$ to the real unit interval $[0,1]$ satisfying the following requirements:

(i) $\Pi(\emptyset)=0$

(ii) for any family $\left\{A_{j}, j \in J\right\}$ of elements of $\mathcal{P}(\mathbf{X})$

$$
\Pi\left(\bigcup_{j \in J} A_{j}\right)=\max _{j \in J} \Pi\left(A_{j}\right) .
$$

$\Pi$ is called normal if $\Pi(\mathbf{X})=1$. Within this paper we will always assume that $\Pi$ is normal. Normality is not only a pleasant technical property, but it also allows a sensible probabilistic interpretation of possibility measures, as we shall see in the next paragraph.

For any $\Pi$ there exists a mapping

$$
\pi: \mathbf{X} \longrightarrow[0,1]
$$

called a distribution of $\Pi$, such that for any $A \in \mathcal{P}(\mathbf{X})$,

$$
\Pi(A)=\max _{x \in A} \pi(x) .
$$

This function is a possibilistic counterpart of a density function in probability theory.

Considering an arbitrary possibility distribution $\pi$ defined on a product universe of discourse $\mathbf{X} \times \mathbf{Y}$, its marginal possibility distribution on $\mathbf{X}$ is defined by the equality

$$
\pi_{X}(x)=\max _{y \in \mathbf{Y}} \pi(x, y)
$$

for any $x \in \mathbf{X}$.

\footnotetext{
${ }^{1}$ In [5] possibility measures are defined in a more general way: instead of the power set $\mathcal{P}(\mathbf{X})$ a complete field is considered and unit interval $[0,1]$ is substituted by a complete lattice.
} 


\subsection{Upper envelopes of sets of probability distributions}

With any possibility measure $\Pi$ on $\mathbf{X}$, one can associate a class of probability measures $\mathcal{M}(\Pi)$ on $\mathbf{X}$ dominated by it, i.e.

$$
\mathcal{M}(\Pi)=\{P: P(A) \leq \Pi(A) \forall A \in \mathcal{P}(\mathbf{X})\},
$$

called a credal set [1].

As proven in 3, the normality of $\Pi$ is equivalent not only to the fact that $\mathcal{M}(\Pi) \neq \emptyset$, but also that $\Pi$ is an upper envelope, i. e. that for any $A \in \mathcal{P}(\mathbf{X})$ there exists $P \in \mathcal{M}(\Pi)$ such that $P(A)=\Pi(A)$.

Nevertheless, within this contribution we will deal with possibility distributions rather than with measures and therefore also with credal sets of probability distributions

$$
\mathcal{M}(\pi)=\{p: p(x) \leq \pi(x) \forall x \in \mathbf{X}\} .
$$

The following example illustrates how $\mathcal{M}(\pi)$ looks like in the most simple, onedimensional case.

Example 2.1. Let $X$ be a binary variable with values in $\mathbf{X}=\{x, \bar{x}\}$ and $\pi(x)=$ $1, \pi(\bar{x})=0.6$ its possibility distribution. Then

$$
\mathcal{M}(\pi)=\{p: p(x)=\alpha \in[0.4,1], p(\bar{x})=1-p(x)\}
$$

is a credal set of probability distributions dominated by $\pi$. As $\pi$ is normal, it is also the upper envelope of $\mathcal{M}(\pi)$.

\section{DECISION-MAKING IN PROBABILITY THEORY}

This section is devoted to basic concepts from decision-making in probabilistic framework.

\subsection{Decision functions and their optimality}

Let $X_{1}, \ldots, X_{n}$ be a set of random variables with values in $\mathbf{X}_{1}, \ldots, \mathbf{X}_{n}\left(\left|\mathbf{X}_{i}\right|<\infty\right.$, $i=1, \ldots, n)$, respectively. A decision function is defined as a mapping

$$
d: \mathbf{X}_{i=1}^{n} \mathbf{X}_{i} \longrightarrow \mathbf{A}
$$

where $\mathbf{A}$ is a finite set of possible alternatives (decisions). Let us assume, at this moment, that we know ${ }^{2}$ the probability distribution $p$ defined on the Cartesian product

$$
\mathbf{X}_{i=1}^{n} \mathbf{X}_{i} \times \mathbf{A}
$$

To simplify the notation we will denote

$$
\mathbf{X}=\mathbf{X}_{i=1}^{n} \mathbf{X}_{i}
$$

\footnotetext{
${ }^{2}$ Decision-making under such considerations is usually called under risk [4].
} 
Let $\mathcal{D}(\mathbf{X}, \mathbf{A})$ denote the set of all decision functions on $\mathbf{X}$ with values in $\mathbf{A}$. If there is no doubt about $\mathbf{X}$ and $\mathbf{A}$, we will simply write $\mathcal{D}$ instead of $\mathcal{D}(\mathbf{X}, \mathbf{A})$.

Let us define an error of decision $d(x)$ (for any $x \in \mathbf{X}$ ) by the equality

$$
e_{p}(d(x) ; x)=\sum_{a \in \mathbf{A}: a \neq d(x)} p(x, a)
$$

and an expected error of decision function $d$ as

$$
e_{p}(d)=\sum_{x \in \mathbf{X}} e_{p}(d(x) ; x)=\sum_{(x, a) \in \mathbf{X} \times \mathbf{A}} p(x, a)(1-\delta(d(x), a)),
$$

where $\delta(u, v)=1$ if $u=v$ and $\delta(u, v)=0$ otherwise.

Our aim is to provide such decisions, which minimize the expected error. Knowing the distribution $p$ we choose a decision function $d_{p}$ satisfying the inequality

$$
e_{p}\left(d_{p}\right) \leq e_{p}(d)
$$

for every decision function $d \in \mathcal{D}(\mathbf{X}, \mathbf{A})$. Function $d_{p}$ is decision function optimal with respect to $p$.

\subsection{Decision-making under uncertainty}

Situations when the precise probability distribution is known are very rare. Usually we only know that the correct distribution $p$ belongs to some class of distributions $\mathfrak{P}$. In these situations different principles (e.g. maximum entropy principle, minimax principle etc.) are applied. In this paper we will deal with minimax principle described bellow.

If we choose decision function $d \in \mathcal{D}(\mathbf{X}, \mathbf{A})$, its expected error can be as large as

$$
\max _{p \in \mathfrak{P}} e_{p}(d)
$$

If we wish this maximum expected error to be the least possible, we have to choose such $d$ that minimizes this expression. Then, we get so-called minimax rule

$$
\min _{d \in \mathcal{D}} \max _{p \in \mathfrak{P}} e_{p}(d)
$$

The decision function optimal with respect to minimax rul $3^{3}$ will be denoted

$$
d^{*} \in \arg \min _{d \in \mathcal{D}} \max _{p \in \mathfrak{P}} e_{p}(d)
$$

In the next part of this paper we will call (1) potential error of decision function $d$ with respect to $\mathfrak{P}$ and denote poterror $(d ; \mathfrak{P})$.

\footnotetext{
${ }^{3}$ Let us note that this decision function need not be unique.
} 


\section{POSSIBILITY DISTRIBUTIONS AND DECISION FUNCTIONS}

As discussed already in Section 2.2 any possibility distribution $\pi$ can be associated with a set of probability distributions $\mathcal{M}(\pi)$ dominated by it. Therefore, minimax principle can be applied to $\mathcal{M}(\pi)$.

\subsection{Possibilistically optimal decision function}

Let us illustrate the application of minimax rule to a credal set $\mathcal{M}(\pi)$ on a simple example.

Example 4.1. Let $\mathbf{X}=\left\{x_{1}, x_{2}\right\}, \mathbf{A}=\left\{a_{1}, a_{2}\right\}$ and the possibility distribution on $\mathbf{X} \times \mathbf{A}$ be defined by Table 1 .

\begin{tabular}{|ll|cc|c|}
\hline$\pi(x, a)$ & & $a_{1}$ & $a_{2}$ & $\pi(x)$ \\
\hline & $x_{1}$ & 1 & .2 & 1 \\
& $x_{2}$ & .5 & .8 & .8 \\
\hline$\pi(a)$ & & 1 & .8 & \\
\hline
\end{tabular}

Tab. 1. Possibility distribution on $\mathbf{X} \times \mathbf{A}$.

This possibility distribution is upper envelope of the credal set contained in Table 2 , where $\alpha, \beta \in[0,0.8], \gamma \in[\max (0, \alpha-0.5, \beta-0.2), \min (\beta, \alpha)]$.

\begin{tabular}{|cc|cc|c|}
\hline$p(x, a)$ & & $a_{1}$ & $a_{2}$ & $p(x)$ \\
\hline & $x_{1}$ & $1-\alpha-\beta+\gamma$ & $\beta-\gamma$ & $1-\alpha$ \\
& $x_{2}$ & $\alpha-\gamma$ & $\gamma$ & $\alpha$ \\
\hline$p(a)$ & & $1-\beta$ & $\beta$ & \\
\hline
\end{tabular}

Tab. 2. Probability distributions on $\mathbf{X} \times \mathbf{A}$ dominated by $\pi$.

On $\mathbf{X}$ we can define the following four decision functions:

$$
\begin{array}{ll}
d_{1}\left(x_{1}\right)=a_{1}, & d_{1}\left(x_{2}\right)=a_{1}, \\
d_{2}\left(x_{1}\right)=a_{1}, & d_{2}\left(x_{2}\right)=a_{2}, \\
d_{3}\left(x_{1}\right)=a_{2}, & d_{3}\left(x_{2}\right)=a_{1}, \\
d_{4}\left(x_{1}\right)=a_{2}, & d_{4}\left(x_{2}\right)=a_{2} .
\end{array}
$$

The expected and potential errors of particular decision functions are contained in Table 3. It is easy to realize that the maximum of $d_{1}$ is reached for $\beta=0.8$, while the maximum of $d_{4}$ for $\beta=0$. Function $d_{3}$ achieves its maximum value for $\alpha, \beta, \gamma=0$, therefore it remains to find maximum for $d_{2}$. This function maximizes for any $\alpha \in[0.5,0.8]$, $\beta=\alpha-0.3, \gamma=\alpha-0.5$; in this interval it attains value 0.7 .

Therefore, the optimal decision function with respect to the minimax principle is $d_{2}$. 


\begin{tabular}{|c|c|c|}
\hline Decision & Exp. error & Pot. error \\
\hline$d_{1}$ & $\beta$ & .8 \\
$d_{2}$ & $\alpha+\beta-2 \gamma$ & .7 \\
$d_{3}$ & $1-\alpha-\beta+2 \gamma$ & 1 \\
$d_{4}$ & $1-\beta$ & 1 \\
\hline
\end{tabular}

Tab. 3. Expected and potential errors of decision functions.

The above mentioned idea leads to the following definition of possibilistically optimal decision function.

Definition 4.2. Let $\pi$ be a possibility distribution and $\mathcal{M}(\pi)$ a credal set dominated by $\pi$. We will call decision function $d^{\pi}$ possibilistically optimal if

$$
d^{\pi} \in \arg \min _{d \in \mathcal{D}} \max _{p \in \mathcal{M}(\pi)} e_{p}(d)
$$

To find $d^{\pi}$ means to find maximum of each decision function and then to choose a function (it need not be unique) with the smallest maximum. The first part of this task is rather difficult (as we have already seen even in Example 4.1), because usually we have to find a constrained extreme of each decision function.

To simplify the procedure, let us utilize the fact that we are dealing with special type of credal sets - sets, the upper envelope of which are possibility distributions.

\subsection{Bounds of the error of a decision function}

With any possibility distribution and any decision function we will associate two (generally different) errors: A minimal error of decision function $d$ defined as

$$
\underline{e_{\pi}}(d)=\max _{x \in \mathbf{X}} \max _{a \in \mathbf{A}: a \neq d(x)} \pi(x, a)=\max _{(x, a) \in \mathbf{X} \times \mathbf{A}} \pi(x, a)(1-\delta(d(x), a)),
$$

and a maximal error of decision function $d$ defined by the formula

$$
\overline{e_{\pi}}(d)=\min \left(\sum_{(x, a) \in \mathbf{X} \times \mathbf{A}} \pi(x, a)(1-\delta(d(x), a)), 1\right) .
$$

The following theorem proves the adequacy of the names of these errors.

Theorem 4.3. Let $\pi$ be a possibility distribution on $\mathbf{X} \times \mathbf{A}$ and $\mathcal{M}(\pi)$ be the corresponding credal set. Then:

(i) for any decision function $d: \mathbf{X} \rightarrow \mathbf{A}$

$$
\operatorname{poterror}(d ; \mathcal{M}(\pi)) \in\left[\underline{e_{\pi}}(d), \overline{e_{\pi}}(d)\right],
$$


(ii) if, furthermore, $d \equiv a_{i}$ then

$$
\operatorname{poterror}(d ; \mathcal{M}(\pi))=\max _{a \in \mathbf{A}: a \neq a_{i}} \pi_{\mathbf{A}}(a),
$$

where $\pi_{\mathbf{A}}$ is marginal distribution of $\pi$ on $\mathbf{A}$.

Proof.

(i) First, let us prove that poterror $(d ; \mathcal{M}(\pi)) \geq \underline{e_{\pi}}(d)$. Since $\pi$ is the upper envelope of $\mathcal{M}(\pi)$, then for any value of $\pi(x, a)$ there exists a probability distribution $p^{\prime}$ such that $p^{\prime}(x, a)=\pi(x, a)$. Particularly, it holds also for maximal $\pi(x, a)$ such that $d(x) \neq a$. Therefore,

$$
\begin{aligned}
\underline{e_{\pi}}(d) & =\max _{(x, a) \in \mathbf{X} \times \mathbf{A}} \pi(x, a)(1-\delta(d(x), a))=\max _{(x, a) \in \mathbf{X} \times \mathbf{A}} p^{\prime}(x, a)(1-\delta(d(x), a)) \\
& \leq \sum_{(x, a) \in \mathbf{X} \times \mathbf{A}} p^{\prime}(x, a)(1-\delta(d(x), a)) \leq \operatorname{poterror}(d ; \mathcal{M}(\pi)) .
\end{aligned}
$$

Now, let us prove that $e_{p}(d) \leq \overline{e_{\pi}}(d)$ for any $p \in \mathcal{M}(\pi)$. Any $p(x, a) \leq \pi(x, a)$ and therefore also

$$
\sum_{(x, a) \in \mathbf{X} \times \mathbf{A}} p(x, a)(1-\delta(d(x), a)) \leq \sum_{(x, a) \in \mathbf{X} \times \mathbf{A}} \pi(x, a)(1-\delta(d(x), a)) .
$$

The remaining inequality

$$
\sum_{(x, a) \in \mathbf{X} \times \mathbf{A}} p(x, a)(1-\delta(d(x), a)) \leq 1
$$

is straightforward.

(ii) The expected error of a decision function $d \equiv a_{i}$ is

$$
e_{p}(d)=\sum_{a \in \mathbf{A}: a \neq a_{i}} p(a)=P\left(\mathbf{A} \backslash\left\{a_{i}\right\}\right) .
$$

Its maximum is (since $\pi$ is the upper envelope of $\mathcal{M}(\pi)$ ) equal to

$$
\Pi\left(\mathbf{A} \backslash\left\{a_{i}\right\}\right)=\max _{a \in \mathbf{A}: a \neq a_{i}} \pi(a)
$$

by definition.

From this proposition we immediately get the following corollary.

Corollary 4.4. Let $\pi$ be a possibility distribution on $\mathbf{X} \times \mathbf{A}$ and $\mathcal{M}(\pi)$ be the corresponding credal set. If, furthermore, there exists $(x, a)$ such that $\pi(x, a)=1$ and $d(x) \neq a$, then

$$
\operatorname{poterror}(d, \mathcal{M}(\pi))=1 \text {. }
$$


Proof. The result follows directly from the application of (i) of Theorem 4.3 to $\pi$ (since from $(2)$ we have that $\underline{e_{\pi}}(d)=1$ ).

Although both the proposition and its corollary are rather simple, they are quite helpful, as they allow us to obtain maxima of expected errors of a number of particular decision functions and at least an estimate of the rest of them, which can help us to find the optimal decision function, as can be seen from the following simple example.

Example 4.5. (Example 4.1 continued) Using (ii) of Theorem 4.3 , we get

$$
\operatorname{poterror}\left(d_{1} ; \mathcal{M}(\pi)\right)=\pi\left(a_{2}\right)=0.8
$$

and

$$
\operatorname{poterror}\left(d_{4} ; \mathcal{M}(\pi)\right)=\pi\left(a_{1}\right)=1
$$

(to the same conclusion we would come if we used Corollary 4.4 instead of (ii)). Using Corollary 4.4 of Theorem 4.3 one gets

$$
\operatorname{poterror}\left(d_{3} ; \mathcal{M}(\pi)\right)=1 .
$$

For decision function $d_{2}$ we can use neither (ii) of Theorem 4.3 nor Corollary 4.4 , but from (i) we get

$$
\operatorname{poterror}\left(d_{2} ; \mathcal{M}(\pi)\right) \in\left[\max \left(\pi\left(x_{1}, a_{2}\right), \pi\left(x_{2}, a_{1}\right)\right), \min \left(\pi\left(x_{1}, a_{2}\right)+\pi\left(x_{2}, a_{1}\right), 1\right)\right]
$$

i. e. poterror $\left(d_{2} ; \mathcal{M}(\pi)\right) \in[0.5,0.7]$. Comparing all potential errors (or their bounds), we immediately see that $d^{\pi}=d_{2}$ is the possibilistically optimal decision function (as expected). Therefore, it is not necessary to look for maxima of particular decision functions.

\subsection{Insensitivity of minimax rule in possibilistic framework}

Unfortunately, one substantial problem appears when for one $x \in \mathbf{X}$ there exist two alternatives $a_{i}, a_{j}$, such that $a_{i} \neq a_{j}$ and $\pi\left(x, a_{i}\right)=\pi\left(x, a_{j}\right)=1$, as demonstrated by the following simple example.

Example 4.6. Let $\mathbf{X}=\left\{x_{1}, x_{2}\right\}, \mathbf{A}=\left\{a_{1}, a_{2}\right\}$ and the possibility distribution on $\mathbf{X} \times \mathbf{A}$ be defined by Table 4

\begin{tabular}{|lc|cc|c|}
\hline$\pi(x, a)$ & & $a_{1}$ & $a_{2}$ & $\pi(x)$ \\
\hline & $x_{1}$ & 1 & 1 & 1 \\
& $x_{2}$ & .5 & .8 & .8 \\
\hline$\pi(a)$ & & 1 & 1 & \\
\hline
\end{tabular}

Tab. 4. Possibility distribution on $\mathbf{X} \times \mathbf{A}$. 
This possibility distribution is the upper envelope of the set of probability distributions contained again in Table 2 , but in this case $\alpha \in[0,0.8], \beta \in[0,1]$, $\gamma \in[\max (0, \alpha-0.5, \alpha+\beta-1), \alpha]$.

Let $d_{i}, i=1, \ldots, 4$ be defined as in previous examples. The expected and potential errors of decision functions are contained in Table 5.

\begin{tabular}{|c|c|c|}
\hline Decision & Exp. error & Pot. error \\
\hline$d_{1}$ & $\beta$ & 1 \\
$d_{2}$ & $\alpha+\beta-2 \gamma$ & 1 \\
$d_{3}$ & $1-\alpha-\beta+2 \gamma$ & 1 \\
$d_{4}$ & $1-\beta$ & 1 \\
\hline
\end{tabular}

Tab. 5. Expected and potential errors of decision functions.

Therefore it is evident that we cannot distinguish among these four decision functions.

\section{LOCALLY OPTIMAL DECISION FUNCTIONS}

To solve the above-mentioned problem, let us recall so-called local minimax principle, already defined in [6].

\subsection{Local minimax principle}

Let us again assume that we only know that the correct distribution belongs to some class of distributions $\mathfrak{P}$. If we make a decision $d(x)=a$, the value of the corresponding error can be as large as

$$
\max _{p \in \mathfrak{P}} e_{p}(a ; x) .
$$

If we wish this maximum error to be the least possible one, we have to choose such $a$ that minimizes this expression. Then we get a local minimax rule

$$
\min _{a \in \mathbf{A}} \max _{p \in \mathfrak{P}} e_{p}(a ; x)
$$

Any optimal decision function (again, it need not be unique) with respect to this rule will be denoted $d_{*}$ and called local minimax decision function.

Analogous to Subsection 3.2 we call (3) potential error of decision $a$ in $x$ with respect to $\mathfrak{P}$ and denote poterror $(a ; x ; \mathfrak{P})$.

It is evident that this approach has one big advantage - we need not to compare the maxima of all decision functions, since we construct the optimal decision function step-by-step for each value of $x \in \mathbf{X}$.

\subsection{Error of locally optimal decision function in possibilistic framework}

Let us apply the local minimax principle to situation described in Example 4.6. 
Example 5.1. (Example 4.6 continued) For $x_{1}$ we can choose either $a_{1}$ or $a_{2}$ - in both cases the error can be as large as 1 . If we choose for $x_{2}$ the alternative $a_{1}$ the potential error is 0.8 , while for the alternative $a_{2}$ only 0.5 . Therefore, both $d_{2}$ and $d_{4}$ are locally optimal decision functions with respect to $\mathcal{M}(\pi)$ (but $d_{1}$ and $d_{3}$ are not).

The following theorem will help us to find the optimal decision function with respect to the local minimax principle.

Theorem 5.2. Let $\pi$ be a possibility distribution on $\mathbf{X} \times \mathbf{A}$ and $\mathcal{M}(\pi)$ be the corresponding credal set. Then for any decision $d(x)=a$

$$
\operatorname{poterror}(a ; x ; \mathcal{M}(\pi))=\max _{a_{i} \in \mathbf{A}: a_{i} \neq a} \pi\left(x, a_{i}\right)
$$

Proof. This proposition can be proved completely analogously to (ii) of Theorem 4.3 . The error of decision $d(x)=a$ is

$$
e_{p}(a ; x)=\sum_{a_{i} \in \mathbf{A}: a_{i} \neq a} p\left(a_{i}, x\right)=P(\{x\} \times \mathbf{A} \backslash\{a\}) .
$$

Its maximum is (since $\pi$ is the upper envelope of $\mathcal{M}(\pi)$ ) equal to

$$
\Pi(\{x\} \times \mathbf{A} \backslash\{a\})=\max _{a_{i} \in \mathbf{A}: a_{i} \neq a} \pi\left(x, a_{i}\right)
$$

by definition.

Thanks to Theorem 5.2 we are ready to define the locally optimal decision function of possibility distribution as follows.

Definition 5.3. Let $\pi$ be a possibility distribution and $\mathcal{M}(\pi)$ a credal set dominated by $\pi$. We will call decision function $d_{\pi}$ possibilistically locally optimal if for any $x \in \mathbf{X}$

$$
d_{\pi}(x) \in \arg \max _{a \in \mathbf{A}} \pi(x ; a) .
$$

Therefore, it is extremely simple to find possibilistically locally optimal decision function: for each $x$ we choose such $a$ for which $\pi(x, a)$ is maximal.

\subsection{Relationship between $d^{\pi}$ and $d_{\pi}$}

Let us note, that there is no direct relationship between errors and expected errors of "usual" and locally optimal minimax decision functions. It means that a function optimal with respect to minimax rule need not be optimal with respect to local minimax rule and vice versa as can be seen from the following simple example.

Example 5.4. Let $\mathbf{X}=\left\{x_{1}, x_{2}\right\}, \mathbf{A}=\left\{a_{1}, a_{2}\right\}$ and the possibility distribution on $\mathbf{X} \times \mathbf{A}$ be defined by Table 6 .

This possibility distribution is the upper envelope of the set of probability distributions contained again in Table 2 in this case $\alpha, \beta \in[0,0.8], \gamma \in[\max (0, \alpha-0.5, \alpha+\beta-$ $1), \alpha]$. 


\begin{tabular}{|ll|cc|c|}
\hline$\pi(x, a)$ & & $a_{1}$ & $a_{2}$ & $\pi(x)$ \\
\hline & $x_{1}$ & 1 & .8 & 1 \\
& $x_{2}$ & .5 & .8 & .8 \\
\hline$\pi(a)$ & & 1 & .8 & \\
\hline
\end{tabular}

Tab. 6. Possibility distribution on $\mathbf{X} \times \mathbf{A}$.

\begin{tabular}{|c|c|c|}
\hline Decision & Exp. error & Pot. error \\
\hline$d_{1}$ & $\beta$ & .8 \\
$d_{2}$ & $\alpha+\beta-2 \gamma$ & 1 \\
$d_{3}$ & $1-\alpha-\beta+2 \gamma$ & 1 \\
$d_{4}$ & $1-\beta$ & 1 \\
\hline
\end{tabular}

Tab. 7. Expected and potential errors of decision functions.

On $\mathbf{X}$ we can define four decision functions with values in $\mathbf{A}$ as in previous examples. The expected and potential errors of particular decision functions are contained in Table 7. It is easy to realize that possibilistically optimal decision function $d^{\pi}$ is $d_{1}$.

On the other hand, using local minimax principle, we get $d_{\pi}\left(x_{1}\right)=a_{1}$ (since by Theorem 5.2

$$
\begin{aligned}
0.8 & =\operatorname{poterror}\left(a_{1} ; x_{1} ; \mathcal{M}(\pi)\right) \\
& \left.\leq \operatorname{poterror}\left(a_{2} ; x_{1} ; \mathcal{M}(\pi)\right)=1\right)
\end{aligned}
$$

and $d_{\pi}\left(x_{2}\right)=a_{2}($ since

$$
\begin{aligned}
0.5 & =\operatorname{poterror}\left(a_{2} ; x_{2} ; \mathcal{M}(\pi)\right) \\
& \leq \operatorname{poterror}\left(a_{1} ; x_{2} ; \mathcal{M}(\pi)\right)=0.8
\end{aligned}
$$

by the same theorem). Therefore, $d_{\pi}=d_{2}$.

\section{CONCLUSIONS}

In this paper, we dealt with decision-making in possibility theory. We presented an approach alternative to that introduced by Kramosil [5], based on a simple idea that any possibility distribution can be associated with a credal set of probability distributions dominated by it. To this credal set minimax principle can be applied and a decision function optimal with respect to it is called possibilistically optimal decision function.

However, since minimax principle is not able to distinguish, in some cases, among decision functions, in particular within the framework of possibility theory, we recalled also local minimax principle introduced already in [6] and realized that it has three advantages in comparison with "classical" minimax principle: 
- it is, in general, much simpler to construct the optimal decision function (step-bystep for each value of domain);

- it is possible to better distinguish among decision functions, at least in possibility theory;

- it is extremely simple to find possibilistically optimal decision function.

\section{ACKNOWLEDGEMENTS}

The support of Grant No. GAČR 13-20012S is gratefully acknowledged.

(Received June 20, 2014)

\section{REFERENCES}

[1] F. G. Cozman: Sets of probability distributions, independence, and convexity. Synthese 186 (2012), 577-600. DOI:10.1007/s11229-011-9999-0

[2] G. de Cooman: Possibility theory I-III. Int. J. General Systems 25 (1997), 291-371. DOI:10.1080/03081079708945160

[3] G. de Cooman and D. Aeyels: Supremum preserving upper probabilities. Inform. Sci. 118 (1999), 173-212. DOI:10.1016/s0020-0255(99)00007-9

[4] K. J. Engemann and R. R. Yager: A general approach to decision making with interval probabilities. Int. J. General Systems 30 (2001), 623-647. DOI:10.1080/03081070108960738

[5] I. Kramosil: Decision-making under uncertainty processed by lattice/valued possibilistic measures. Kybernetika 42 (2006), 629-646.

[6] J. Vejnarová: On various criteria of optimality in probabilistic decision-making. Kybernetika 28 (1992), 5, 392-401.

Jirina Vejnarová, Institute of Information Theory and Automation - Academy of Sciences of the Czech Republic, Pod Vodárenskou věži 4, 18208 Praha 8. Czech Republic.

e-mail:vejnar@utia.cas.cz 